\title{
Histological Study of Discoid Lateral Meniscus in Children and Adolescents: Morphogenetic Considerations
}

\author{
Cosimo Tudisco $^{1}$ Flavia Botti ${ }^{2}$ Salvatore Bisicchia ${ }^{1}$ \\ ${ }^{1}$ Department of Orthopaedic Surgery, University of Rome "Tor \\ Vergata," Rome, Italy \\ 2 Department of Clinical Sciences and Translational Medicine, \\ University of Rome "Tor Vergata", Rome, Italy \\ Address for correspondence Cosimo Tudisco, MD, Department of \\ Orthopaedic Surgery, Viale Oxford 81, 00133 Rome, Italy \\ (e-mail: cosimo.tudisco@uniroma2.it).
}

Joints 2019;7:155-158.

\begin{abstract}
Background Discoid lateral meniscus is the most frequent variant of the meniscus. Few studies have focused on the histology of discoid menisci. The aim of the present study was to report the histological findings of discoid lateral meniscus in children and adolescents, after arthroscopic partial resection, to give a possible explanation of its developmental etiology.

Methods Five patients aged 9,10,13,15, and 17 years were operated on for a 1-piece excision of a discoid lateral meniscus, and the specimens were histologically examined. Results The extracellular matrix showed a different distribution and characteristics depending on the different side of the meniscus. Irregularly oriented collagen fibers in discoid lateral meniscus were found. Cells of different shapes were observed depending on the surficial or deep location in the tissue. There were no blood vessels in the inner

Keywords

- discoid lateral meniscus

- histology

- children

- knee

- development part of discoid lateral meniscus.

Conclusion The findings of the current study seem to confirm that discoid lateral meniscus arises from variant morphogenesis. Furthermore, the altered distribution and shape of the cells and disorganization of collagen fibers (irrespectively of the age of the patients) may predispose discoid lateral meniscus to degeneration, damage, and tear in young patients also.

Level of Evidence Level of evidence 4 (case series).
\end{abstract}

\section{Introduction}

Discoid lateral meniscus is the most frequent variant of the meniscus and it is bilateral in up to $20 \%$ of cases. ${ }^{1}$ Discoid menisci can be classified according to Watanabe et $\mathrm{al}^{2}$ in complete, incomplete, and Wrisberg's variants. A ringshaped variant was subsequently described. ${ }^{3}$
There is still debate about the etiology of discoid meniscus. Smillie ${ }^{4}$ first proposed that it is normally present at some stages during fetal development. However, most of the authors believe that it is anomalous also during prenatal development and arises through variant morphogenesis., 6 In fact, it is frequently associated to other abnormalities, often an incidental finding, and commonly asymptomatic. ${ }^{1}$ received

March 7, 2018

accepted

April 18, 2021

published online

June 18, 2021
DOI https://doi.org/

10.1055/s-0041-1730979.

ISSN 2282-4324.

\footnotetext{
(C) 2021. The Author(s).

This is an open access article published by Thieme under the terms of the Creative Commons Attribution-NonDerivative-NonCommercial-License, permitting copying and reproduction so long as the original work is given appropriate credit. Contents may not be used for commercial purposes, or adapted, remixed, transformed or built upon. (https://creativecommons.org/ licenses/by-nc-nd/4.0/) Georg Thieme Verlag KG, Rüdigerstraße 14, 70469 Stuttgart, Germany
} 
Although the histology of normal menisci in children and in adults has been well described, ${ }^{2}$ few studies focused on the histology of discoid menisci, unfortunately including a great number of adults and meniscal tears. ${ }^{7-10}$

The aim of the present study was to report the histological findings of discoid lateral meniscus in children and adolescents, after arthroscopic partial resection, to give a possible explanation of its developmental etiology.

\section{Methods}

This study is conformed to the Declaration of Helsinki as revised in 2000. Approval from the Institutional Review Board was waived for this study at the author's Institution. Parents of the young patients signed a written informed consent prior to surgery, authorizing histological examination of the specimens.

From 2012 to 2016, all the children and adolescents presenting for a symptomatic (locking, caching, popping, and/or pain) discoid lateral meniscus at the authors' Institution were prospectively scrutinized and enrolled in the study. There were no exclusion criteria for this study.

Five patients were arthroscopically operated on for a symptomatic discoid lateral meniscus at our Department. During each intervention, partial meniscectomy (saucerization) with preservation of a stable peripheral rim was performed to obtain the shape of a normal meniscus. The resected tissue was decalcified in ethylenediaminetretracetic acid (EDTA) buffered for 2 hours, fixed in $4 \%$ buffered formaldehyde for 24 hours, and placed in a series of graded ethanol solution for paraffin embedding. From the paraffin blocks, 3- $\mu \mathrm{m}$ thick sections were cut both parallel and perpendicular to the articular horizontal plane and stained with hematoxylin and eosin, Masson trichromic, and AlcianPeriodic acid-Schiff reaction (PAS). The sections were examined with an Axioskop microscope (Carl Zeiss Light Microscopy, Göttingen, Germany) and photographed with a digital camera (Rt Slider, Diagnostic Instrument Inc., Germany).

\section{Results}

There were one female and four male patients aged 9, 10, 13, 15 , and 17 years. The right knee was involved in two cases. At preoperative magnetic resonance imaging (MRI) and at arthroscopic examination, a complete variant was diagnosed. ${ }^{2}$

At full-thickness sections cut perpendicular to the surface, the tibial surface appeared mainly smooth, while the femoral surface appeared undulated, frayed or in some cases with clefts; the extracellular matrix, showed a lower intensity of staining, on the periphery of both the tibial and femoral surfaces. The decreasing cell gradient of the normal meniscus was completely absent ( $\mathbf{- F i g . 1} \mathbf{1}$ ).

The collagen fibers in the inner part showed a woven arrangement, while peripheral parts were larger and formed a tighter network (-Fig. 2). Several tears were observed (asterisks in - Fig. 1). Degenerated extracellular matrix with disorganized and less-dense collagen fibers was observed in some areas of the different sections. (-Figs. 1-4). On both

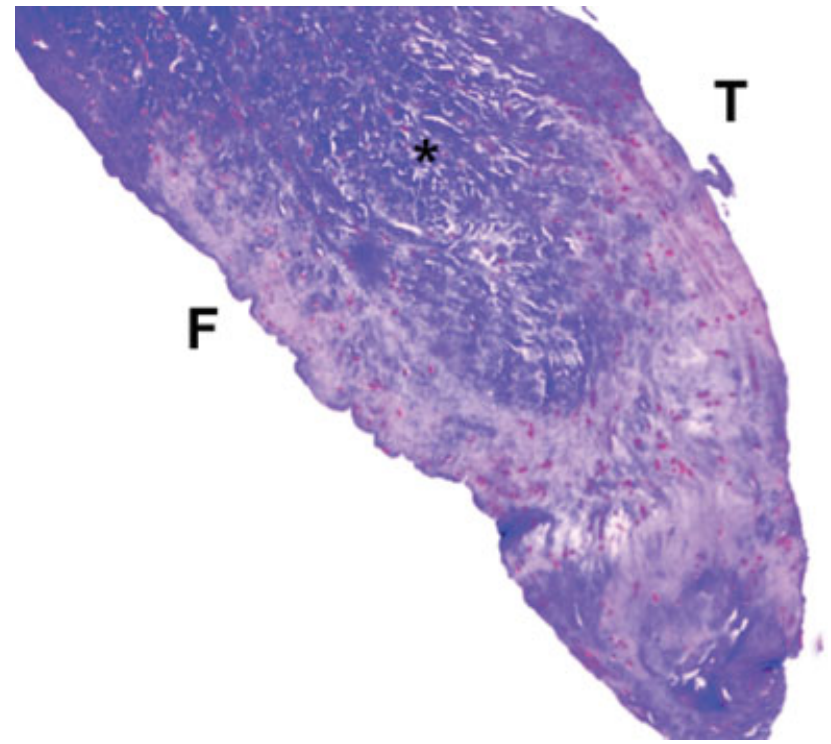

Fig. 1 Section perpendicular to the articular surfaces of a discoid lateral meniscus in a 13-year-old patient ( $\times 5$ magnification, Masson's trichrome). The femoral surface $(F)$ shows an irregular and notched profile unlike the tibial surface $(T)$ which appears smooth and more regular. Within the fibrocartilaginous tissue, areas of wide tissue degeneration are evident, represented both by a lower intensity of staining and by the presence of completely disorganized collagen fibers. There are also areas of tissue lacerations associated with a lower cell density (asterisks). It is possible to observe the different aspect of the cell component. On the articular sides, cells have a more flattened aspect while in the innermost portion the cells appear more rounded.

meniscal surfaces, cells had a more flattened, fusiform morphology (similar to fibroblasts). In contrast, the central portion cell was more rounded (similar to chondrocytes; -Figs. 1 and 2). The vascular supply was completely absent in all specimens.

Both on the tibial and femoral side, cells were randomly scattered, the collagen fibers were irregularly represented, with a loose texture. Some areas of hypocellularity as a sign of degeneration were present ( - Fig. 4).

On sections cut parallel to the articular surface, thick bundles of almost homogeneous size, with variable directions, were observed, cells had a round morphology, and a random distribution (- Fig. 4). A lower intensity of staining, with disorganized and inhomogeneous aspect, was observed in some areas, with collagen fibers distributed without a definite arrangement. Cells were decreased and irregularly distributed (-Figs. 4 and $\mathbf{5}$ ).

\section{Discussion}

The histologic findings of our study seem to confirm that discoid lateral meniscus is different from a normal meniscus in terms of distribution and shape of the cells, vascularity, and organization of collagen fibers, irrespectively of the age of the patients.

Discoid meniscus represents the most common congenital abnormality of the knee meniscus. Cadaveric studies reported the prevalence of discoid lateral meniscus to be between 0 and 7\%, while arthroscopic studies reported a prevalence from 0.4 to $16.6 \%,{ }^{11}$ with the highest values in the 


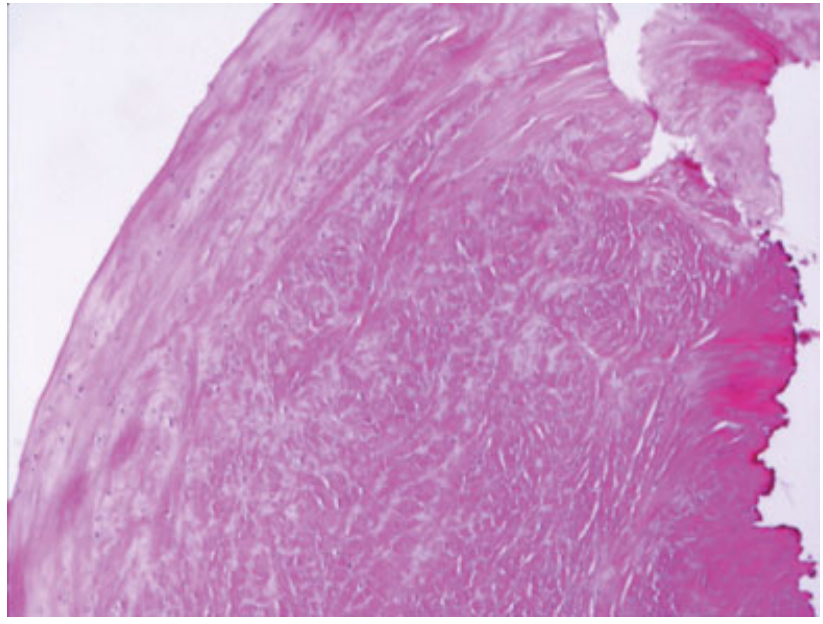

Fig. 2 Section perpendicular to the articular surfaces of a discoid lateral meniscus in a 13-year-old patient ( $\times 10$ magnification, hematoxylin and eosin). This picture shows how the thickness and orientation of the collagen fibers vary from the surface to the innermost portion of the tissue. On the surface, we observe thick fibers with parallel orientation to the surface. Moving toward the center, we observe collagen fibers with random orientation and considerably reduced thickness. Edematous areas were also evident in the tissue.

Asian population (20\%). ${ }^{1}$ The exact pathologic alterations and cause of this discoid shape is still debated. Smillie, ${ }^{4}$ who proposed the first theory about the discoid meniscus, considered that it resulted from the persistence of a normal fetal stage of normal development, consequence of a failure of resorption of the central area of a cartilaginous disc. Kaplan ${ }^{6}$ refuted this theory and proposed a developmental theory, considering the discoid shape to be the consequence of the abnormal meniscal motion due to the lack of attachment to the tibia, and definitely revealed that the menisci are never discoid during the development. This last conclusion was confirmed also by Clark and Ogden ${ }^{5}$ in their prenatal and postnatal cadaver knee studies of the developmental changes

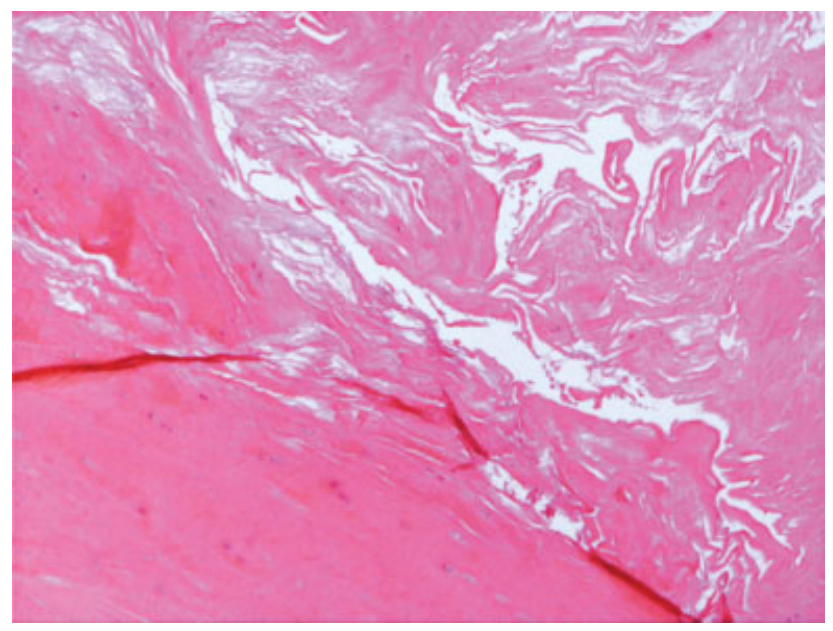

Fig. 3 Section perpendicular to the articular surfaces of a discoid lateral meniscus in a 17-year-old patient $(\times 20$ magnification, hematoxylin and eosin). This section shows a markedly degenerated fibrocartilaginous tissue with large areas of tissue breakdown. Note the complete absence of the cellular component in the most degenerated areas.

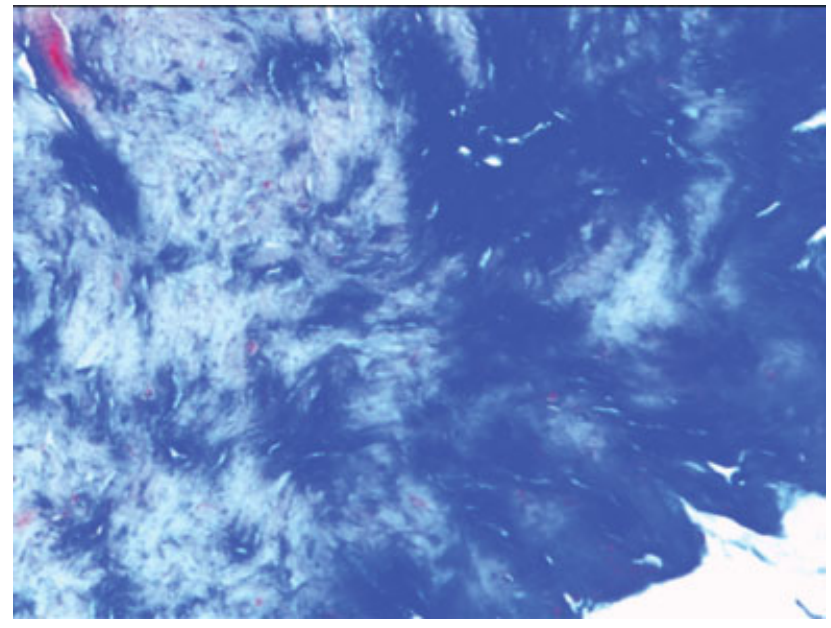

Fig. 4 Section parallel to the articular surfaces of a discoid lateral meniscus in a 17-year-old patient ( $\times 10$ magnification Masson's trichrome). Tissue degeneration is even more marked here, especially in the innermost portion of the meniscus. Edema associated with a poor presence of the cellular component and an important alteration of the extracellular matrix are evident in this section.

that occur in the menisci prior to skeletal maturity. Le Minor $^{12}$ reported also that no embriological study in humans had ever shown a discoid shape in lateral menisci, and concluded that discoid lateral meniscus does not originate from a persistence of normal embryonic structure. Murlimanju et al, $^{13}$ studying the lateral meniscus of the knee in South Indian human fetuses, reported an incidence of a discoid-shaped lateral meniscus in only $17.9 \%$ of the cases, while the majority of the knees had a nondiscoid shape. They concluded that the lateral meniscus is anomalous and arises from variant morphogenesis.

Despite the large number of anatomic, clinical, and arthroscopic studies on discoid lateral meniscus, there are limited data in the literature regarding its histology that can help to understand its possible developmental etiology.

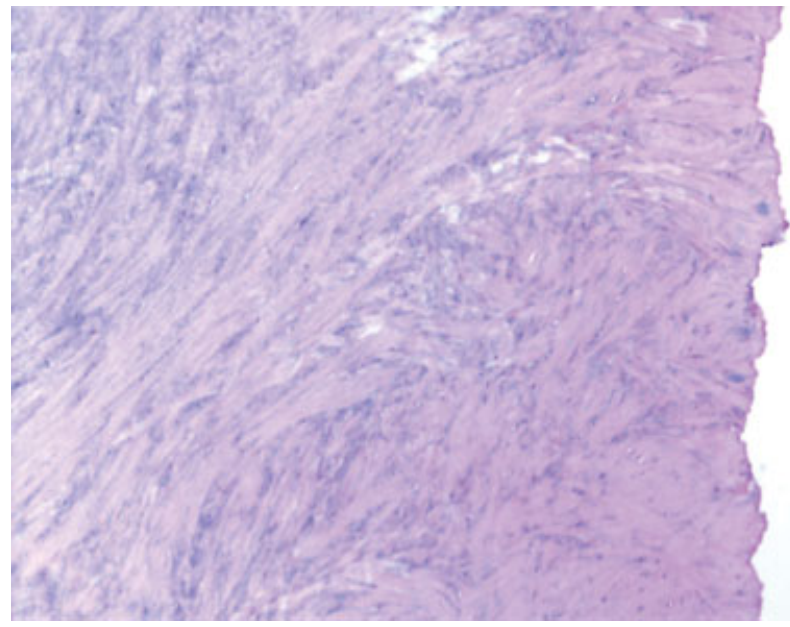

Fig. 5 Section parallel to the articular surfaces in a 17-year-old patient ( $\times 10$ magnification, Masson's trichrome). In this image it is possible to observe the presence of collagen fibers with a different orientation and a cell component randomly scattered inside the different areas of the tissue. 
Only few papers described the histology of discoid meniscus. Atay et $\mathrm{al}^{7}$ reported a decrease in the number of collagen fibers with a heterogeneous course in discoid menisci. Cui and $\mathrm{Min}^{9}$ reported the femoral surface of discoid meniscus to be covered by dense and well-arranged thick fibrils, while the tibial surface to have an irregular orientation of fibers. This significant disorganization of the circular collagen network in the discoid meniscus with a heterogeneous course of the circumferentially arranged collagen fibers with areas of degeneration was confirmed by Papadopoulos et al. ${ }^{8}$ He concluded that the discoid meniscus represents a structural lesion rather than a morphologic variant.

The limitations of those studies are the inclusion of a great percentage of adults and meniscal tears.

The main finding of the current study is the description of the histologic features of discoid lateral meniscus in a group composed only by children and adolescents without a meniscal tear. In our study, the significant disorganization of the circular collagen network in the discoid meniscus with a heterogeneous course of the circumferentially arranged collagen fibers was observed, together with areas of degeneration with lower density of extracellular matrix, evidenced by a lower intensity of staining.

Most authors also believe that the discoid meniscus must be of congenital origin, and support that the congenital theory comes from evidence of familial transmission and reports of occurrence in twins. Discoid meniscus has also been described associated with agenesis of anterior cruciate ligament, ${ }^{14}$ with cord-like anterior intermeniscal ligament, ${ }^{15}$ and with hypoplasia of the medial femoral condyle. ${ }^{16}$ These last reports seem to confirm that the discoid lateral meniscus is a complete different entity from the normal meniscus with a possible genetic transmission, and not a consequence of an altered meniscal development. Furthermore, regrowth of a discoid lateral meniscus after arthroscopic reshaping has been described in an adolescent, as to indicate the genetic information to obtain the discoid shape at the end of skeletal growth. ${ }^{17}$

We observed also a scattered distribution of the cells in the discoid meniscus. They appeared also very different depending on where they were observed in the tissue: from a very flat shape close to the outer part of the meniscus to an oval/round shape (like chondrocytes) in the inner part. These histological observations have never been described in a normal meniscus.

All our samples were obtained at surgery from children or adolescent patients. We did not find differences in the different samples examined at different ages.

These findings suggest that discoid lateral meniscus is different from a normal meniscus in terms of cellularity and organization of collagen fibers. We speculate that the disorganization of collagen is a native feature of discoid lateral meniscus, and not a consequence of the degeneration induced by the altered mechanics. Thus, we agree with Murlimanju et $\mathrm{al}^{13}$ that the discoid lateral meniscus must be anomalous and due to a variant morphogenesis.

\section{Limitations}

The main limitation of the current study is the lack of comparison with a normal meniscus, but there were no meniscal tears in children and adolescents during the study period that required resection (we usually perform meniscal repair in these young patients).

\section{Conclusion}

In conclusion, the histologic findings of our study seem to confirm that discoid lateral meniscus is different from a normal meniscus in terms of distribution and shape of the cells, vascularity, and organization of collagen fibers, irrespective of the patients' age. This features may predispose discoid lateral meniscus to degeneration, damage, and tear in young patients also.

\section{Conflict of Interest}

None declared.

\section{References}

1 Yaniv M, Blumberg N. The discoid meniscus.J Child Orthop 2007;1 (02):89-96

2 Watanabe M, Takeda S, Ikeuchi H. Atlas of arthroscopy. 3rd ed. Heidelberg, Germany: Springer; 1979

3 Monllau JC, León A, Cugat R, Ballester J. Ring-shaped lateral meniscus. Arthroscopy 1998;14(05):502-504

4 Smillie IS. The congenital discoid meniscus. J Bone Joint Surg $\mathrm{Br}$ 1948;30B(04):671-682

5 Clark CR, Ogden JA. Development of the menisci of the human knee joint. Morphological changes and their potential role in childhood meniscal injury. J Bone Joint Surg Am 1983;65(04):538-547

6 Kaplan EB. Discoid lateral meniscus of the knee joint; nature, mechanism, and operative treatment. J Bone Joint Surg Am 1957; 39-A(01):77-87

7 Atay OA, Pekmezci M, Doral MN, Sargon MF, Ayvaz M, Johnson DL. Discoid meniscus: an ultrastructural study with transmission electron microscopy. Am J Sports Med 2007;35(03):475-478

8 Papadopoulos A, Kirkos JM, Kapetanos GA. Histomorphologic study of discoid meniscus. Arthroscopy 2009;25(03):262-268

9 Cui JH, Min BH. Collagenous fibril texture of the discoid lateral meniscus. Arthroscopy 2007;23(06):635-641

10 Choi YH, Seo YJ, Ha JM, Jung KH, Kim J, Song SY. Collagenous ultrastructure of the discoid meniscus: a transmission electron microscopy study. Am J Sports Med 2017;45(03):598-603

11 Kelly BT, Green DW. Discoid lateral meniscus in children. Curr Opin Pediatr 2002;14(01):54-61

12 Le Minor JM. Comparative morphology of the lateral meniscus of the knee in primates. J Anat 1990;170:161-171

13 Murlimanju BV, Nair N, Ray B, Pai MM, Amin S, Pai SR. Morphological variants of lateral meniscus of the knee: a cadaveric study in South Indian human fetuses. Anat Sci Int 2011;86(02):63-68

14 Rayar M, Bouillis J, Fraisse B, Marleix S, Chapuis M, Violas P. Discoid meniscus associated with agenesis of the anterior cruciate ligament in an 8-year-old child. Orthop Traumatol Surg Res 2011;97(08):874-876

15 Ozcanli H, Keles N, Gocmen-Mas N, Ozenci AM, Aydin AT. Relation of discoid lateral meniscus and cord-like anterior intermeniscal ligament: morphological and clinical study. Surg Radiol Anat 2011;33(08):673-678

16 Wang HD, Gao SJ. Bilateral discoid medial meniscus associated with meniscal tears and hypoplasia of the medial femoral condyle: a case report. Medicine (Baltimore) 2017;96(46):e8637

17 Bisicchia S, Tudisco C. Re-growth of an incomplete discoid lateral meniscus after arthroscopic partial resection in an 11 year-old boy: a case report. BMC Musculoskelet Disord 2013;14:285 